\title{
Formulation and Solution of Curved Beams with Elastic Supports
}

\author{
Fernando SARRIA, Faustino N. GIMENA, Pedro GONZAGA, Mikel GOÑI, Lázaro GIMENA
}

\begin{abstract}
This article presents the general system of differential equations that governs the behaviour of a curved beam, which can be solved by either numerical or analytical methods. The obtained solution represents the matricial expression of transference. The stiffness matrix is derived directly rearranging the transfer matrix. Through twelve equations are shown the elastic conditions of the support in both ends of the curved piece. By joining the twelve equations of the stiffness matrix expression with the twelve equations of support conditions, we determined a unique system of equations associated to the curved beam with elastic supports. Establishing the elastic conditions has always been a problem, since previous traditional models do not look at the whole system, of twenty four equations, with all the unknowns and all the functions. Two examples of pieces with elastic supports are developed to show the applicability of the proposed method.
\end{abstract}

Keywords: analytical and numerical solutions; curved beam; stiffness matrix; support equations; transfer matrix

\section{INTRODUCTION}

In order to solve the problem of a system of linear ordinary differential equations with boundary conditions, numerical and analytic strategies were used. Approximate procedures [1] have been used since and exact analytical solution is normally very difficult to obtain. Some examples of numerical methods to solve these boundary value problems are: the Finite Differences [2], Boundary Element [3], Finite Element [4], Shooting method [5] and Differential Quadrature methods [6]. There is extensive literature on modelling arbitrary curved beam elements [7-11]. Conventionally, the laws governing the mechanical behaviour of a curved warped beam (applying the Euler-Bernoulli and Timoshenko theories) are defined by static equilibrium and kinematics [12-13] or dynamic motion equations [14]. A few authors present this definition with compact energy equations [15-17]. These interpretations have allowed reaching accurate results for some types of beams: for instance, a circular arch element loaded in plane [18-22] and loaded perpendicular to its plane [23], elliptical and parabolic beams loaded in plane [24-27] or a helix uniformly loaded [28]. This paper presents the general formulation of a curved beam [29], which is solved by an analytical procedure or by a numerical procedure called Finite Transfer Method [30]. The two ends of a curved beam are connected through the expression obtained using any of the previous procedures, analytic or numeric. An algebraic equation system of dimension $p$ is obtained. The formulation of the problem is completed when the $p$ elastic supports conditions are incorporated. Then a final algebraic system of $2 p$ order is solved. The values at any point of the domain can be determined, once the values at the initial point are known. Two examples are given to show the procedure exposed that is good in the practice.

\section{FORMULATION AND SOLUTION OF THE CURVED BEAM}

A curved beam is formed by a plane cross section, whose centroid sweeps through all the points of an axis curve. The vector radius expresses the curved line:

$$
\boldsymbol{r}=\boldsymbol{r}(s)
$$

where $s$ is the independent variable (arc length of the centroid line). Its unit vectors tangent $\boldsymbol{t}(s)$, normal $\boldsymbol{n}(s)$ and binormal $\boldsymbol{b}(s)$ are:

$$
\begin{aligned}
& \boldsymbol{t}(s)=\operatorname{Dr}(s) \\
& \boldsymbol{n}(s)=D^{2} \boldsymbol{r}(s) /\left|D^{2} \boldsymbol{r}(s)\right| \\
& \boldsymbol{b}(s)=\boldsymbol{t}(s) \wedge \boldsymbol{n}(s)
\end{aligned}
$$

where $D=\mathrm{d} / \mathrm{d} s$ is the derivative with respect to the variable $s$.

The natural equations of the centroid line [31] are expressed by the flexion and torsion curvatures:

$$
\begin{aligned}
& \chi(s)=\sqrt{D^{2} \boldsymbol{r}(s) \cdot D^{2} \boldsymbol{r}(s)} \\
& \tau(s)=\operatorname{Dr}(s) \cdot \frac{\left(D^{2} \boldsymbol{r}(s) \wedge D^{3} \boldsymbol{r}(s)\right)}{\left(D^{2} \boldsymbol{r}(s) \cdot D^{2} \boldsymbol{r}(s)\right)}
\end{aligned}
$$

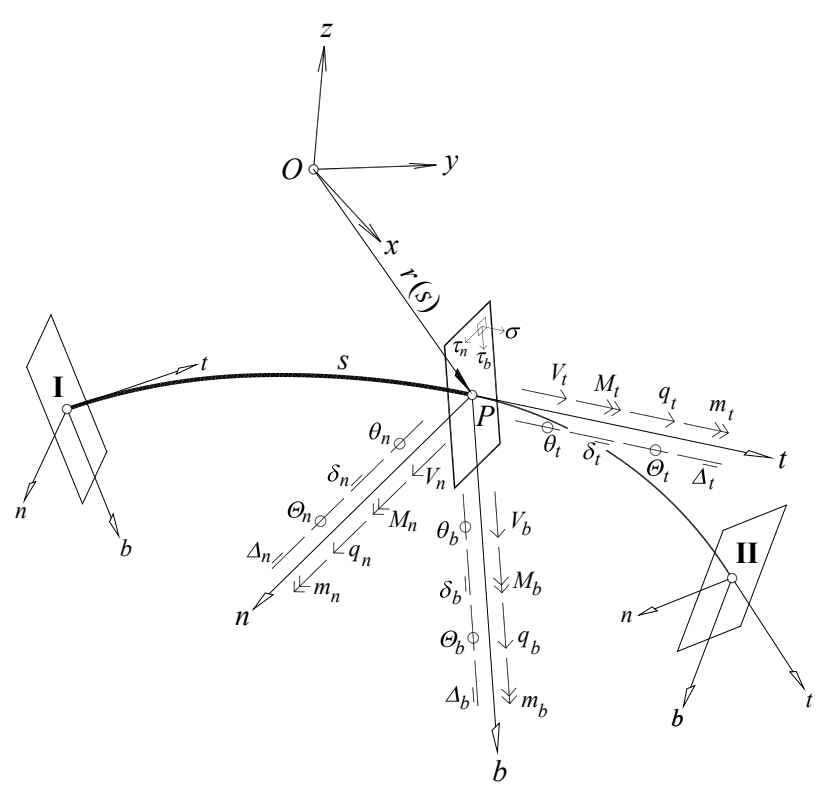

Figure 1 Curved beam with state and load vectors

The Frenet-Serret frame $P_{t n b}$ (Fig. 1) is the reference coordinate system used here to represent the intervening known and unknown functions of the problem. 


\subsection{Formulation: Differential System}

When we assume the habitual hypotheses and principles, Euler-Bernoulli and Timoshenko classical beam theories [12, 32], and consider the stresses associated with the normal cross-section $\sigma, \tau_{n}, \tau_{b}$ the geometric characteristics of the section are: shearing coefficients $\alpha_{n}, \alpha_{n b}, \alpha_{b}$, area $A$ and moments of inertia $I_{t}$, $I_{n}, I_{b}, I_{n b}$. Transversal $G$ and longitudinal $E$ elasticity moduli provide the elastic properties of the material.

The system of differential equations governing the structural behaviour of a spatially curved beam can be obtained by applying kinematics and equilibrium laws to an infinitesimal element of the curve $[29,33]$ :

$$
\begin{aligned}
& D V_{t}-\chi V_{n} \\
& \begin{aligned}
\chi V_{t}+D V_{n} & -\tau V_{b} \\
\tau V_{n} & +D V_{b}
\end{aligned}
\end{aligned}
$$

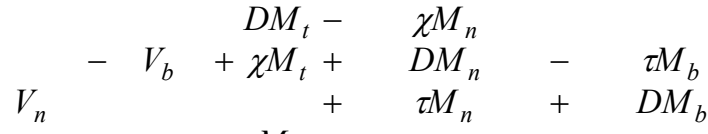

$$
\begin{aligned}
& -\frac{M_{t}}{G I_{t}} \\
& +D \theta_{t}-\chi \theta_{n} \\
& +q_{t}=0 \\
& +q_{n}=0 \\
& +q_{b}=0 \\
& +m_{t}=0 \\
& +m_{n}=0 \\
& +m_{b}=0 \\
& -\Theta_{t}=0 \\
& -\frac{I_{b} M_{n}}{E\left[I_{n} I_{b}-I_{n b}^{2}\right]}-\frac{I_{n b} M_{b}}{E\left[I_{n} I_{b}-I_{n b}^{2}\right]}+\chi \theta_{t}+D \theta_{n}-\theta_{b} \\
& -\Theta_{n}=0 \\
& -\frac{I_{n b} M_{n}}{E\left[I_{n} I_{b}-I_{n b}^{2}\right]}-\frac{I_{n} M_{b}}{E\left[I_{n} I_{b}-I_{n b}^{2}\right]}+\tau \theta_{n}+D \theta_{b} \\
& -\Theta_{b}=0 \\
& -\frac{V_{t}}{E A} \\
& +D \delta_{t}-\chi \delta_{n} \quad-\Delta_{t}=0 \\
& -\frac{\alpha_{n} V_{n}}{G A}-\frac{\alpha_{n b} V_{b}}{G A} \\
& -\theta_{b}+\chi \delta_{t}+D \delta_{n}-\delta_{b}-\Delta_{n}=0 \\
& -\frac{\alpha_{n b} V_{n}}{G A}-\frac{\alpha_{b} V_{b}}{G A} \\
& +\theta_{n} \quad+\tau \delta_{n}+D \delta_{b}-\Delta_{b}=0
\end{aligned}
$$

The equilibrium equations are represented by the first six rows of the differential system, Eq. (4). The functions involved in it are (Fig. 1):

Moments and internal forces

$$
\begin{aligned}
& {\left[\begin{array}{l}
\boldsymbol{V} \\
\boldsymbol{M}
\end{array}\right]=\left[\begin{array}{ccc}
V_{t} & V_{n} & V_{b} \\
M_{t} & M_{n} & M_{b}
\end{array}\right]\left[\begin{array}{l}
\boldsymbol{t} \\
\boldsymbol{n} \\
\boldsymbol{b}
\end{array}\right]=} \\
& =\left[\begin{array}{ccc}
\int_{A} \sigma \mathrm{d} A & \int_{A} \tau_{n} \mathrm{~d} A & \int_{A} \tau_{b} \mathrm{~d} A \\
\int_{A}\left(\tau_{b} n-\tau_{n} b\right) \mathrm{d} A & \int_{A} \sigma b \mathrm{~d} A & -\int_{A} \sigma n \mathrm{~d} A
\end{array}\right]\left[\begin{array}{l}
\boldsymbol{t} \\
\boldsymbol{n} \\
\boldsymbol{b}
\end{array}\right]
\end{aligned}
$$

Load forces-moments

$$
\left[\begin{array}{c}
\boldsymbol{q}_{\boldsymbol{V}} \\
\boldsymbol{q}_{\boldsymbol{M}}
\end{array}\right]=\left[\begin{array}{ccc}
q_{t} & q_{n} & q_{b} \\
m_{t} & m_{n} & m_{b}
\end{array}\right]\left[\begin{array}{l}
\boldsymbol{t} \\
\boldsymbol{n} \\
\boldsymbol{b}
\end{array}\right]
$$

The kinematics equations are represented by the last six rows of the differential system, Eq. (4). The functions involved in the kinematics equations are (Fig. 1):

Rotations and displacements

$$
\left[\begin{array}{l}
\boldsymbol{\theta} \\
\boldsymbol{\delta}
\end{array}\right]=\left[\begin{array}{ccc}
\theta_{t} & \theta_{n} & \theta_{b} \\
\delta_{t} & \delta_{n} & \delta_{b}
\end{array}\right]\left[\begin{array}{l}
\boldsymbol{t} \\
\boldsymbol{n} \\
\boldsymbol{b}
\end{array}\right]
$$

Load rotation-displacement
$\left[\begin{array}{l}\boldsymbol{q}_{\boldsymbol{\Theta}} \\ \boldsymbol{q}_{\boldsymbol{\Delta}}\end{array}\right]=\left[\begin{array}{lll}\Theta_{t} & \Theta_{n} & \Theta_{b} \\ \Delta_{t} & \Delta_{n} & \Delta_{b}\end{array}\right]\left[\begin{array}{l}\boldsymbol{t} \\ \boldsymbol{n} \\ \boldsymbol{b}\end{array}\right]$

The differential system, Eq. (4) may also be expressed in the vector-matrix form as follows:

$\operatorname{De}(s)=[\boldsymbol{T}(s)] \boldsymbol{e}(s)+\boldsymbol{q}(s)$

where, $\boldsymbol{e}(s)$ is the state vector of internal forces and deflections at a point $s$ of the beam element, which is named Effect at the Section

$$
\boldsymbol{e}(s)=\{\boldsymbol{V}, \boldsymbol{M}, \boldsymbol{\theta}, \boldsymbol{\delta}\}^{\mathrm{T}}
$$

$q(s)$ is the Applied Load

$$
\boldsymbol{q}(s)=\left\{-\boldsymbol{q}_{\boldsymbol{V}},-\boldsymbol{q}_{\boldsymbol{M}}, \boldsymbol{q}_{\boldsymbol{\theta}}, \boldsymbol{q}_{\boldsymbol{\delta}}\right\}^{\mathrm{T}}
$$

and $[\boldsymbol{T}(s)]$ is the Infinitesimal Transfer Matrix

$$
[\boldsymbol{T}(s)]=\left[\begin{array}{cccc}
{[D]} & {[\boldsymbol{0}]} & {[\boldsymbol{0}]} & {[\boldsymbol{0}]} \\
{[\psi]} & {[D]} & {[\boldsymbol{0}]} & {[\boldsymbol{0}]} \\
{[\boldsymbol{0}]} & -\left[\theta_{\boldsymbol{M}}\right] & {[D]} & {[\boldsymbol{0}]} \\
-\left[\delta_{\boldsymbol{V}}\right] & {[\boldsymbol{0}]} & {[\psi]} & {[D]}
\end{array}\right]
$$

In this last expression, the matrices that are involved are: 
the Frenet-Serret

$[D]=\left[\begin{array}{ccc}0 & \chi(s) & 0 \\ -\chi(s) & 0 & \tau(s) \\ 0 & -\tau(s) & 0\end{array}\right]$

of moments produced by forces and displacements produced by rotations

$[\psi]=\left[\begin{array}{ccc}0 & 0 & 0 \\ 0 & 0 & -1 \\ 0 & 1 & 0\end{array}\right]$

rotations produced by moments

$\left[\theta_{M}\right]=\left[\begin{array}{ccc}\frac{1}{G I_{t}} & 0 & 0 \\ 0 & \frac{I_{b}}{E\left[I_{n} I_{b}-I_{n b}^{2}\right]} & \frac{I_{n b}}{E\left[I_{n} I_{b}-I_{n b}^{2}\right]} \\ 0 & \frac{I_{n b}}{E\left[I_{n} I_{b}-I_{n b}^{2}\right]} & \frac{I_{n}}{E\left[I_{n} I_{b}-I_{n b}^{2}\right]}\end{array}\right]$

and displacements produced by forces

$\left[\delta_{V}\right]=\left[\begin{array}{ccc}\frac{1}{E A} & 0 & 0 \\ 0 & \frac{\alpha_{n}}{G A} & \frac{\alpha_{n b}}{G A} \\ 0 & \frac{\alpha_{n b}}{G A} & \frac{\alpha_{b}}{G A}\end{array}\right]$

An alternate approach expressed in the global Cartesian coordinate system is given by [33].

\subsection{Analytical Solution}

The exact analytical solution of the system expressed in Eq. (9) is given by [30]:

$\boldsymbol{e}(s)=\left[\boldsymbol{T}\left(s_{\boldsymbol{I}}, s\right)\right] \boldsymbol{e}\left(s_{\boldsymbol{I}}\right)+\boldsymbol{q}\left(s_{\boldsymbol{I}}, s\right)$

where, $\left[\boldsymbol{T}\left(s_{I}, s\right)\right]$ is the Transfer Matrix from initial $\boldsymbol{I}$ to a general point $s$

$$
\left[\boldsymbol{T}\left(s_{\boldsymbol{I}}, s\right)\right]=e^{\int_{S_{\boldsymbol{I}}}^{s}[\boldsymbol{T}(s)] \mathrm{d} s}
$$

$\boldsymbol{q}(S I, S)$ is the Load Transmitted from initial $\boldsymbol{I}$ to a general point $s$

$\boldsymbol{q}\left(s_{\boldsymbol{I}}, s\right)=e^{\int_{S_{\boldsymbol{I}}}^{s}[\boldsymbol{T}(s)] \mathrm{d} s} \int_{s_{\boldsymbol{I}}}^{s} \boldsymbol{q}(s) e^{-\int_{S_{\boldsymbol{I}}}^{s}[\boldsymbol{T}(s)] \mathrm{d} s} \mathrm{~d} s$

Previous solution particularised for both extremes $I$ and $\boldsymbol{I I}$ of the curved beam gives the following relation:
$\boldsymbol{e}\left(s_{\boldsymbol{I I}}\right)=\left[\boldsymbol{T}\left(s_{\boldsymbol{I}}, s_{\boldsymbol{I I}}\right)\right] \boldsymbol{e}\left(s_{\boldsymbol{I}}\right)+\boldsymbol{q}\left(s_{\boldsymbol{I}}, s_{\boldsymbol{I I}}\right)$

where $\left[\boldsymbol{T}\left(s_{\boldsymbol{I}}, s_{\boldsymbol{I I}}\right)\right]$ is the Exact Analytical Transfer Matrix

$\left[T\left(s_{I}, s_{I I}\right)\right]=\left[\begin{array}{cccc}{\left[T_{V_{I}}^{V_{I I}}\right]} & {[0]} & {[0]} & {[0]} \\ {\left[T_{V_{I}}^{M_{I I}}\right]} & {\left[T_{M_{I}}^{M_{I I}}\right]} & {[0]} & {[0]} \\ {\left[T_{V_{I}}^{\theta_{I I}}\right]} & {\left[T_{M_{I}}^{\theta_{I I}}\right]} & {\left[T_{\theta_{I}}^{\theta_{I I}}\right]} & {[0]} \\ {\left[T_{V_{I}}^{\delta_{I I}}\right]} & {\left[T_{M_{I}}^{\delta_{I I}}\right]} & {\left[T_{\theta_{I}}^{\delta_{I I}}\right]} & {\left[T_{\delta_{I}}^{\delta_{I I}}\right]}\end{array}\right]$

$\boldsymbol{q}\left(s_{\boldsymbol{I}}, s_{I I}\right)$ is the Load Transfer Vector

$\boldsymbol{q}\left(s_{I}, s_{I I}\right)=\left\{\boldsymbol{q}_{V_{I, I I}}, \boldsymbol{q}_{M_{I, I I}}, \boldsymbol{q}_{\boldsymbol{\theta}_{I, I I}}, \boldsymbol{q}_{\delta_{I, I I}}\right\}^{\mathrm{T}}$

\subsection{Numerical Solution}

The numerical solution to Eq. (9) using the finite transfer method [34] is expressed by the next equation:

$\tilde{\boldsymbol{e}}\left(s_{i+1}\right)=\left[\boldsymbol{T}_{\boldsymbol{F T M}}\left(s_{\boldsymbol{I}}, s_{i+1}\right)\right] \tilde{\boldsymbol{e}}\left(s_{\boldsymbol{I}}\right)+\boldsymbol{q}_{\boldsymbol{F T M}}\left(s_{\boldsymbol{I}}, s_{i+1}\right)=$

$=\left[\prod_{j=0}^{j=i}\left[\boldsymbol{T}_{\boldsymbol{F T M}}\left(s_{j}\right)\right]\right] \tilde{\boldsymbol{e}}\left(s_{\boldsymbol{I}}\right)+\sum_{j=0}^{j=i}\left[\prod_{k=j+1}^{k=i}\left[\boldsymbol{T}_{\boldsymbol{F T M}}\left(s_{k}\right)\right]\right] \boldsymbol{q}_{\boldsymbol{F T M}}\left(s_{j}\right)$

where,

$\left[\boldsymbol{T}_{\boldsymbol{F T M}}\left(s_{j}\right)\right]=[\boldsymbol{I}]+\left[\left[\boldsymbol{T}_{j+1}\right]+4\left[\boldsymbol{T}_{j+1 / 2}\right]+\left[\boldsymbol{T}_{j}\right]\right] \Delta s / 6+$

$+\left[\left[\boldsymbol{T}_{j+1}\right]\left[\boldsymbol{T}_{j+1 / 2}\right]+\left[\boldsymbol{T}_{j+1 / 2}\right]^{2}+\left[\boldsymbol{T}_{j+1 / 2}\right]\left[\boldsymbol{T}_{j}\right]\right] \Delta s^{2} / 6+$

$+\left[\left[\boldsymbol{T}_{j+1}\right]\left[\boldsymbol{T}_{j+1 / 2}\right]^{2}+\left[\boldsymbol{T}_{j+1 / 2}\right]^{2}\left[\boldsymbol{T}_{j}\right]\right] \Delta s^{3} / 12+$

$+\left[\boldsymbol{T}_{j+1}\right]\left[\boldsymbol{T}_{j+1 / 2}\right]^{2}\left[\boldsymbol{T}_{j}\right] \Delta s^{4} / 24$

$\boldsymbol{q}_{\boldsymbol{F T M}}\left(s_{j}\right)=\left(\boldsymbol{q}_{j+1}+4 \boldsymbol{q}_{j+1 / 2}+\boldsymbol{q}_{j}\right) \Delta s / 6+$

$+\left(\left[\boldsymbol{T}_{j+1}\right] \boldsymbol{q}_{j+1 / 2}+\left[\boldsymbol{T}_{j+1 / 2}\right] \boldsymbol{q}_{j+1 / 2}+\left[\boldsymbol{T}_{j+1 / 2}\right] \boldsymbol{q}_{j}\right) \Delta s^{2} / 6+$

$+\left(\left[\boldsymbol{T}_{j+1}\right]\left[\boldsymbol{T}_{j+1 / 2}\right] \boldsymbol{q}_{j+1 / 2}+\left[\boldsymbol{T}_{j+1 / 2}\right]^{2} \boldsymbol{q}_{j}\right) \Delta s^{3} / 12+$

$+\left[\boldsymbol{T}_{j+1}\right]\left[\boldsymbol{T}_{j+1 / 2}\right]^{2} \boldsymbol{q}_{j} \Delta s^{4} / 24$

Assuming the next approximations:

$\mathrm{d} s \cong \Delta s$ and $\boldsymbol{e}\left(s_{i+1}\right)=\tilde{\boldsymbol{e}}\left(s_{i+1}\right)$.

When we establish $n$ intervals, both end points I and II of the curved line can be related:

$\tilde{\boldsymbol{e}}\left(s_{I I}\right)=\left[\boldsymbol{T}_{F T M}\right] \tilde{\boldsymbol{e}}\left(s_{I}\right)+\boldsymbol{q}_{F T M}=$

$=\left[\prod_{j=0}^{j=n-1}\left[\boldsymbol{T}_{\boldsymbol{F T M}}\left(s_{j}\right)\right]\right] \tilde{\boldsymbol{e}}\left(s_{\mathrm{I}}\right)+\sum_{j=0}^{j=n-1}\left[\prod_{k=j+1}^{k=n-1}\left[\boldsymbol{T}_{\boldsymbol{F T M}}\left(s_{k}\right)\right]\right] \boldsymbol{q}_{\boldsymbol{F T M}}\left(s_{j}\right)$ 
where $\left[\boldsymbol{T}_{\boldsymbol{F T M}}\right]$ is the Numerical Transfer Matrix and $\boldsymbol{q}_{\text {FTM }}$ the Load Transfer Vector.

The Numerical Solution is a direct translation of the Analytical as was demonstrated [30]:

$$
\begin{aligned}
& \tilde{\boldsymbol{e}}\left(s_{\boldsymbol{I}}\right)=\boldsymbol{e}\left(s_{\boldsymbol{I}}\right), \tilde{\boldsymbol{e}}\left(s_{\boldsymbol{I I}}\right)=\boldsymbol{e}\left(s_{\boldsymbol{I I}}\right),\left[\boldsymbol{T}_{\boldsymbol{F T M}}\right] \cong\left[\boldsymbol{T}\left(s_{\boldsymbol{I}}, s_{\boldsymbol{I I}}\right)\right] \text { and } \\
& \boldsymbol{q}_{\boldsymbol{F T M}} \cong \boldsymbol{q}\left(s_{\boldsymbol{I}}, s_{\boldsymbol{I I}}\right) .
\end{aligned}
$$

\subsection{Stiffness Matrix}

Rearranging the terms of the transfer equation (Eq. (20) or Eq. (24)) as was presented in [35], the stiffness matrix expression is obtained:

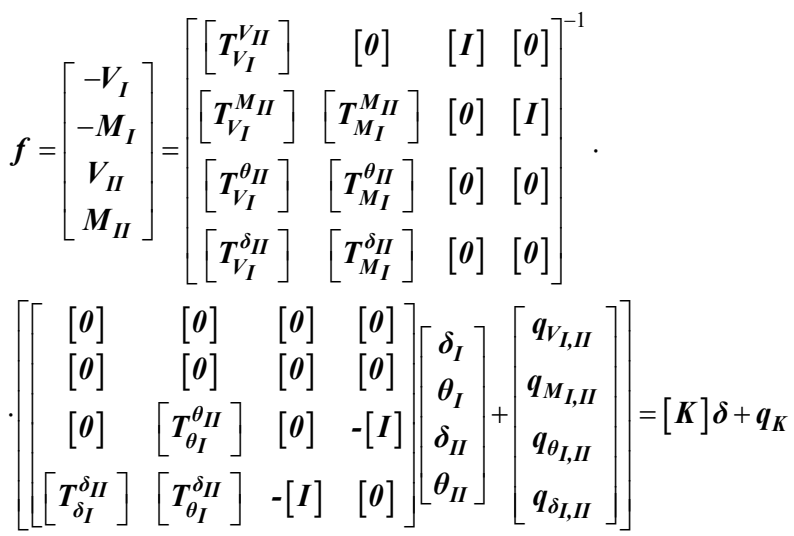

where $f$ and $\delta$ vectors of reactions and displacements unknowns at both ends, $[\boldsymbol{K}]$ the Stiffness Matrix

$$
[K]=\left[\begin{array}{llll}
{\left[K_{V_{I}}^{\delta_{I}}\right]} & {\left[K_{V_{I}}^{\theta_{I}}\right]} & {\left[K_{V_{I}}^{\delta_{I I}}\right]} & {\left[K_{V_{I}}^{\theta_{I I}}\right]} \\
{\left[K_{M_{I}}^{\delta_{I}}\right]} & {\left[K_{M_{I}}^{\theta_{I}}\right]} & {\left[K_{M_{I I}}^{\delta_{I I}}\right]} & {\left[K_{K_{I I}}^{\theta_{I I}}\right]} \\
{\left[K_{V_{I I}}^{\delta_{I}}\right]} & {\left[K_{V_{I I}}^{\theta_{I}}\right]} & {\left[K_{V_{I I}}^{\delta_{I I}}\right]} & {\left[K_{V_{I I}}^{\theta_{I I}}\right]} \\
{\left[K_{M_{I I}}^{\delta_{I}}\right]} & {\left[K_{M_{I I}}^{\theta_{I}}\right]} & {\left[K_{M_{I I}}^{\delta_{I I}}\right]} & {\left[K_{M_{I I}}^{\theta_{I I}}\right]}
\end{array}\right]
$$

and $\boldsymbol{q}_{\boldsymbol{K}}$ the Equivalent Load Vector

$$
\boldsymbol{q}_{K}=\left\{\boldsymbol{q}_{V_{I, I I}}^{K}, \boldsymbol{q}_{M_{I, I I}}^{K}, \boldsymbol{q}_{\boldsymbol{\theta}_{I, I I}}^{K}, \boldsymbol{q}_{\delta_{I, I I}}^{K}\right\}^{\mathrm{T}}
$$

Note that, generally, the isolated beam has not been supported yet, therefore there are twenty four unknowns: twelve of forces and moments and the other twelve of rotations and displacements:

$$
\left[\begin{array}{ll}
{[I]} & -[K] \\
\hdashline[0] & {[0]}
\end{array}\right]\left[\begin{array}{l}
f \\
\delta
\end{array}\right]=\left[\begin{array}{l}
q_{K} \\
-0
\end{array}\right]
$$

It is very practical to write the whole algebraic system with all the intervening unknowns in a single vector in this form since it will be easier to implement the elastic conditions, as will be presented later.

\section{ELASTIC SUPPORT EQUATIONS}

In this section the equations concerning elastic supports are developed. First, the equations associated to the initial support are formulated and then the equations of the final support.

\subsection{Initial Support}

Compatibility and equilibrium relations applied in displacements and forces at the elastic initial support end of the beam:

$\left[[I]-\left[\alpha_{V_{I}}^{\delta_{I}}\right]\right]\left(V_{I}+Q_{I}\right)=\left[K_{V_{I}}^{\delta_{I}} \delta_{i}^{j}\right]\left[\alpha_{V_{I}}^{\delta_{I}}\right]\left(\delta_{I}-\Delta_{I}\right)$

where $\left[\boldsymbol{\alpha}_{\boldsymbol{V}_{\boldsymbol{I}}}^{\boldsymbol{\delta}_{\boldsymbol{I}}}\right]$ is the matrix of the displacements factors of restraints at the elastic support in the initial point

$\left[\boldsymbol{\alpha}_{V_{\boldsymbol{I}}}^{\boldsymbol{\delta}_{\boldsymbol{I}}}\right]=\left[\begin{array}{ccc}\alpha_{V_{t \boldsymbol{I}}}^{\delta_{\boldsymbol{I}}} & 0 & 0 \\ 0 & \alpha_{V_{n \boldsymbol{I}}}^{\delta_{n \boldsymbol{I}}} & 0 \\ 0 & 0 & \alpha_{V_{b \boldsymbol{I}}}^{\delta_{b \boldsymbol{I}}}\end{array}\right]$

$\boldsymbol{Q}_{\boldsymbol{I}}$ is the punctual forces loads applied

$\boldsymbol{Q}_{\boldsymbol{I}}=\left\{Q_{t}^{\boldsymbol{I}}, Q_{n}^{\boldsymbol{I}}, Q_{b}^{\boldsymbol{I}}\right\}^{\mathrm{T}}$

$\boldsymbol{\Lambda}_{\boldsymbol{I}}$ is the imposed (if applied) punctual displacement at the initial point

$\Lambda_{I}=\left\{\Lambda_{t}^{I}, \Lambda_{n}^{I}, \Lambda_{b}^{I}\right\}^{\mathrm{T}}$

and $\delta_{i}^{j}$ is the Kronecker's delta.

When the support has a rigidity that restrains the longitudinal displacement $K_{V_{t}}^{\boldsymbol{I}} \delta_{t}$ its factor can be determined by the next expression:

$\alpha_{V_{t I}}^{\delta_{t} \boldsymbol{I}}=\frac{K_{V_{t} \delta_{t}}^{\boldsymbol{I}}}{K_{V_{t} \delta_{t}}^{\boldsymbol{I}}-K_{V_{t} \delta_{t}}^{\boldsymbol{I}}}$

The rest of $\alpha$ factors can be obtained in the same way.

The rotation for the elastic support relation at the initial point may be noted as:

$\left[[I]-\left[\boldsymbol{\alpha}_{M_{I}}^{\theta_{I}}\right]\right]\left(M_{I}+\Pi_{I}\right)=\left[K_{M_{I}}^{\theta_{I}} \delta_{i}^{j}\right]\left[\boldsymbol{\alpha}_{M_{I}}^{\theta_{I}}\right]\left(\theta_{I}-O_{I}\right)$

where $\left[\boldsymbol{\alpha}_{\boldsymbol{M}_{\boldsymbol{I}}}^{\boldsymbol{\theta}_{\boldsymbol{I}}}\right]$ is the matrix of the rotation factors of restraints at the elastic support in the initial point 
$\left[\boldsymbol{\alpha}_{\boldsymbol{M}_{\boldsymbol{I}}}^{\theta_{\boldsymbol{I}}}\right]=\left[\begin{array}{ccc}\alpha_{M_{t \boldsymbol{I}}}^{\theta_{\boldsymbol{I}}} & 0 & 0 \\ 0 & \alpha_{M_{n \boldsymbol{I}}}^{\theta_{n \boldsymbol{I}}} & 0 \\ 0 & 0 & \alpha_{M_{b \boldsymbol{I}}}^{\theta_{b \boldsymbol{I}}}\end{array}\right]$

$\boldsymbol{\Pi}_{\boldsymbol{I}}$ is the punctual moment actions exerted

$$
\boldsymbol{\Pi}_{\boldsymbol{I}}=\left\{\Pi_{t}^{\boldsymbol{I}}, \Pi_{n}^{\boldsymbol{I}}, \Pi_{b}^{\boldsymbol{I}}\right\}^{\mathrm{T}}
$$

and $\boldsymbol{O}_{\boldsymbol{I}}$ is the imposed (when applied) punctual displacements at the initial point

$\boldsymbol{O}_{\boldsymbol{I}}=\left\{O_{t}^{\boldsymbol{I}}, O_{n}^{\boldsymbol{I}}, O_{b}^{\boldsymbol{I}}\right\}^{\mathrm{T}}$

\subsection{Final Support}

Similarly, extreme elastic conditions can be derivate for the final end of the spatially curved beam. First, on displacements and forces:

$$
-\left[[I]-\left[\boldsymbol{\alpha}_{V_{I I}}^{\delta_{I I}}\right]\right]\left(V_{I I}-Q_{I I}\right)=\left[K_{V_{I I}}^{\delta_{I I}} \delta_{i}^{j}\right]\left[\boldsymbol{\alpha}_{V_{I I}}^{\delta_{I I}}\right]\left(\delta_{I I}-\Delta_{I I}\right)(38
$$

Where $\left[\boldsymbol{\alpha}_{\boldsymbol{V}_{\boldsymbol{I I}}}^{\boldsymbol{\delta}_{\boldsymbol{I I}}}\right]$ is the matrix of the displacement factors of restraints at the elastic support in the final point

$$
\left[\boldsymbol{\alpha}_{\boldsymbol{V}_{\boldsymbol{I I}}}^{\boldsymbol{\delta}_{\boldsymbol{I I}}}\right]=\left[\begin{array}{ccc}
\alpha_{V_{t I \boldsymbol{I I}}}^{\delta_{t I I}} & 0 & 0 \\
0 & \alpha_{V_{n \boldsymbol{I I I}}}^{\delta_{n I I}} & 0 \\
0 & 0 & \alpha_{V_{b I I}}^{\delta_{b I I}}
\end{array}\right]
$$

$\boldsymbol{Q}_{\boldsymbol{I I}}$ is the punctual forces loads applied

$$
\boldsymbol{Q}_{\boldsymbol{I I}}=\left\{Q_{t}^{\boldsymbol{I I}}, Q_{n}^{\boldsymbol{I I}}, Q_{b}^{\boldsymbol{I I}}\right\}^{\mathrm{T}}
$$

and $\Lambda_{I I}$ is the imposed (when applied) punctual displacements at the final point

$$
\Lambda_{I I}=\left\{\Lambda_{t}^{I I}, \Lambda_{n}^{I I}, \Lambda_{b}^{I I}\right\}^{\mathrm{T}}
$$

Secondly, with respect to the rotations we obtain:

$$
-\left[[I]-\left[\boldsymbol{\alpha}_{M_{I I}}^{\theta_{I I}}\right]\right]\left(M_{I I}-\Pi_{I I}\right)=\left[K_{M_{I I}}^{\theta_{I I}} \delta_{i}^{j}\right]\left[\boldsymbol{\alpha}_{M_{I I}}^{\theta_{I I}}\right]\left(\theta_{I I}-O_{I I}\right)
$$

where $\left[\boldsymbol{\alpha}_{\boldsymbol{M}_{\boldsymbol{I I}}}^{\theta_{I I}}\right]$ is the matrix of the rotation factors of restraints at the elastic support in the final extreme

$\left[\boldsymbol{\alpha}_{\boldsymbol{M}_{\boldsymbol{I I}}}^{\theta_{\boldsymbol{I I}}}\right]=\left[\begin{array}{ccc}\alpha_{M_{t I I}}^{\theta_{t I I}} & 0 & 0 \\ 0 & \alpha_{M_{n I I}}^{\theta_{n I I}} & 0 \\ 0 & 0 & \alpha_{M_{b I I}}^{\theta_{b I I}}\end{array}\right]$
$\boldsymbol{\Pi}_{\boldsymbol{I I}}$ is the punctual forces moment applied

$$
\boldsymbol{\Pi}_{\boldsymbol{I I}}=\left\{\Pi_{t}^{I I}, \Pi_{n}^{I I}, \Pi_{b}^{I I}\right\}^{\mathrm{T}}
$$

and $\boldsymbol{O}_{\boldsymbol{I I}}$ is the imposed (when applied) punctual rotations at the final point.

$$
\boldsymbol{O}_{\boldsymbol{I I}}=\left\{O_{t}^{I I}, O_{n}^{\boldsymbol{I I}}, O_{b}^{\text {II }}\right\}^{\mathrm{T}}
$$

If we join previous Eqs. (29), (34), (38) and (42) in a single matrix equation, we will reach the following expression of elastic stiffness conditions:

$$
-[[\boldsymbol{I}]-[\alpha]](\boldsymbol{f}-\boldsymbol{Q})=\left[\boldsymbol{K} \delta_{i}^{j}\right][\alpha](\delta-\boldsymbol{\Delta})
$$

where $[\boldsymbol{\alpha}]$ is the restraints support matrix

$$
[\alpha]=\left[\begin{array}{cccc}
{\left[\alpha_{V_{I}}^{\delta_{I}}\right]} & {[0]} & {[0]} & {[0]} \\
{[0]} & {\left[\alpha_{M_{I}}^{\theta_{I}}\right]} & {[0]} & {[0]} \\
{[0]} & {[0]} & {\left[\alpha_{V_{I I}}^{\delta_{I I}}\right]} & {[0]} \\
{[0]} & {[0]} & {[0]} & {\left[\alpha_{M_{I I}}^{\theta_{I I}}\right.}
\end{array}\right]
$$

$\boldsymbol{Q}$ is the complete vector of forces and moments

$\boldsymbol{Q}=\left\{\boldsymbol{Q}_{\boldsymbol{I}}^{\mathrm{T}}, \boldsymbol{\Pi}_{\boldsymbol{I}}^{\mathrm{T}}, \boldsymbol{Q}_{\boldsymbol{I I}}^{\mathrm{T}}, \boldsymbol{\Pi}_{\boldsymbol{I I}}^{\mathrm{T}}\right\}^{\mathrm{T}}$

and $\boldsymbol{\Lambda}$ is the punctual loads of rotations and displacements in both ends

$$
\boldsymbol{\Lambda}=\left\{\boldsymbol{\Lambda}_{\boldsymbol{I}}^{\mathrm{T}}, \boldsymbol{O}_{\boldsymbol{I}}^{\mathrm{T}}, \boldsymbol{\Lambda}_{\boldsymbol{I I}}{ }^{\mathrm{T}}, \boldsymbol{O}_{\boldsymbol{I I}}{ }^{\mathrm{T}}\right\}^{\mathrm{T}}
$$

Former equation Eq. (46), may be noted as:

$$
\left[\begin{array}{cc}
{[0]} & {[0]} \\
{\left[[\boldsymbol{I}]-[\boldsymbol{\alpha}]\left[\boldsymbol{K} \delta_{i}^{j}\right][\boldsymbol{\alpha}]\right.}
\end{array}\right]\left[\begin{array}{l}
\boldsymbol{f} \\
\boldsymbol{\delta}
\end{array}\right]=\left[\begin{array}{c}
\boldsymbol{0} \\
\hdashline[\boldsymbol{I}]-[\boldsymbol{\alpha}]] \boldsymbol{Q}+\left[\boldsymbol{K} \delta_{i}^{j}\right][\boldsymbol{\alpha}] \boldsymbol{\Lambda}
\end{array}\right]
$$

As shown in section 2.4, where the stiffness matrix was developed and demonstrated in a special way, it is crucial to write the whole algebraic system with all the intervening unknowns in a single vector, because it will be solved with one single stage without adding sophisticated and artificial procedures. This method is easier and more flexible in order to implement the elastic conditions, and has several advantages comparing to other methods used.

\section{CURVED BEAM'S FULL SYSTEM OF EQUATIONS}

Equation Eq. (25) cosiders twelve algebraic equations, which relate displacements and reactions in both ends of the beam. The other twelve equations that are necessary to solve the problem are provided by the elastic condition of the support in Eq. (46). As above mentioned, we may now directly aggregate the 
expressions derived Eq. (28) and Eq. (50) to obtain the most general system, complete with the twenty four equations, which are noted as follows:

$\left[\begin{array}{cc}{[\boldsymbol{I}]} & -[\boldsymbol{K}] \\ \hdashline[\boldsymbol{I}]-[\boldsymbol{\alpha}] & {\left[\boldsymbol{K} \delta_{i}^{j}\right][\boldsymbol{\alpha}]}\end{array}\right]\left[\begin{array}{l}\boldsymbol{f} \\ \boldsymbol{\delta}\end{array}\right]=\left[\begin{array}{c}\boldsymbol{q}_{\boldsymbol{K}} \\ \hdashline[[\boldsymbol{I}]-[\boldsymbol{\alpha}]] \boldsymbol{Q}+\left[\boldsymbol{K} \delta_{i}^{j}\right][\boldsymbol{\alpha}] \boldsymbol{\Lambda}\end{array}\right]$

The algebraic system may be solved directly by obtaining the inverse of the matrix, given the vector of reactions and displacements straightforward by:

$\left[\begin{array}{l}\boldsymbol{f} \\ \boldsymbol{\delta}\end{array}\right]=\left[\begin{array}{cc}{[\boldsymbol{I}]} & -[\boldsymbol{K}] \\ \hdashline[\boldsymbol{I}]-[\boldsymbol{\alpha}] & {\left[\boldsymbol{K} \delta_{i}^{j}\right][\boldsymbol{\alpha}]}\end{array}\right]^{-1}\left[\begin{array}{c}\boldsymbol{q}_{\boldsymbol{K}} \\ {[[\boldsymbol{I}]-[\boldsymbol{\alpha}]] \boldsymbol{Q}+\left[\boldsymbol{K} \delta_{i}^{j}\right][\boldsymbol{\alpha}] \boldsymbol{\Lambda}}\end{array}\right](52)$

Notwithstanding the singularity of the stiffness matrix, thus with no inverse, if the structure is stable, a solution is always presented because it is a compatibility determinated system.

\section{EXAMPLES}

This section presents two examples to show that this method can approach the calculus of different problems of curved beam with elastic supports. The first case is solved by the analytical procedure and the second by the numerical aforementioned method.

\subsection{Traction-Compression Bar}

The first analysed example is the case of a bar under traction-compression effect. The example is considered to be adequate to show the analytic procedure developed in the article.

Let us take a straight beam with uniform force load and elastic support, as shown in Fig. 2 (it is considered to be of constant section and same material).

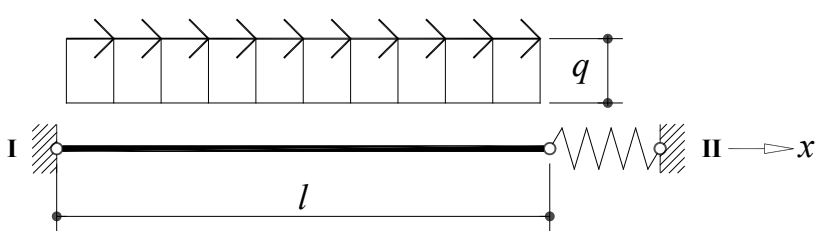

Figure 2 Traction-compression bar with elastic support

The differential system in this case is a particular case of the general Eq. (9) given:

$D V_{x}+q_{x}=0$
$-\frac{V_{x}}{E A}+D \delta_{x}-\Delta_{x}=0 ; q_{x}=q ; \Delta_{x}=0$

Integrating with the only force load applied, we obtain:

$\left[\begin{array}{l}V_{x}(l) \\ \delta_{x}(l)\end{array}\right]=\left[\begin{array}{cc}1 & 0 \\ \frac{1}{E A} & 1\end{array}\right]\left[\begin{array}{l}V_{x}(0) \\ \delta_{x}(0)\end{array}\right]+\left[\begin{array}{c}-q l \\ -\frac{q l^{2}}{2 E A}\end{array}\right]$ which is the expression in transference as per Eq. (20).

Reordering reactions and displacements yields:

$\left[\begin{array}{cc}1 & 0 \\ \frac{1}{E A} & 1\end{array}\right]\left[\begin{array}{c}-V_{x}(0) \\ V_{x}(l)\end{array}\right]=\left[\begin{array}{cc}0 & 0 \\ 1 & -1\end{array}\right]\left[\begin{array}{c}\delta_{x}(0) \\ \delta_{x}(l)\end{array}\right]+\left[\begin{array}{c}-q l \\ -\frac{q l^{2}}{2 E A}\end{array}\right]$

After inverting the former matrix and multiplying, all the unknowns are put in a single vector as in Eq. (28):

$\left[\begin{array}{cc:cc}1 & 0 & -E A / l & E A / l \\ 0 & 1 & E A / l & -E A / l \\ \hdashline 0 & 0 & 0 & 0 \\ 0 & 0 & 0 & 0\end{array}\right]\left[\begin{array}{c}-V_{x}(0) \\ V_{x}(l) \\ \hdashline \delta_{x}(0) \\ \delta_{x}(l)\end{array}\right]=\left[\begin{array}{c}-q l / 2 \\ -q l / 2 \\ \hdashline 0 \\ 0\end{array}\right]$

The support conditions of fixed-fixed $(\alpha=1)$ and fixed-free $(\alpha=0)$ are written:

$\left[\begin{array}{cc:cc}0 & 0 & 0 & 0 \\ 0 & 0 & 0 & 0 \\ \hdashline 0 & 0 & 1 & 0 \\ 0 & 1-\alpha & 0 & \alpha E A / l\end{array}\right]\left[\begin{array}{c}-V_{x}(0) \\ V_{x}(l) \\ \hdashline \delta_{x}(0) \\ \delta_{x}(l)\end{array}\right]=\left[\begin{array}{c}0 \\ 0 \\ -0 \\ 0\end{array}\right]$

Adding these last systems Eq. (56) and Eq. (57) into a single equation, we obtain:

$\left[\begin{array}{cc:cc}1 & 0 & -E A / l & E A / l \\ 0 & 1 & E A / l & -E A / l \\ \hdashline 0 & 0 & 1 & 0 \\ 0 & 1-\alpha & 0 & \alpha E A / l\end{array}\right]\left[\begin{array}{c}-V_{x}(0) \\ V_{x}(l) \\ \hdashline \delta_{x}(0) \\ \delta_{x}(l)\end{array}\right]=\left[\begin{array}{c}-q l / 2 \\ -q l / 2 \\ \hdashline 0 \\ 0\end{array}\right]$

Thus, the solution for the state values in the extremes of the bar $\boldsymbol{I}$ and $\boldsymbol{I I}$ is:

$\left[\begin{array}{c}-V_{x}(0) \\ V_{x}(l) \\ \hdashline \delta_{x}(0) \\ \delta_{x}(l)\end{array}\right]=\left[\begin{array}{c}-(2-\alpha) q l / 2 \\ -\alpha q l / 2 \\ ---q \\ 0 \\ (1-\alpha) q l^{2} / 2 E A\end{array}\right]$

Once the initial values are known, the solution at any point is given by:

$$
\begin{gathered}
V_{x}(\xi)=\eta(\xi) \frac{q l}{2}=(2-2 \xi-\alpha) \frac{q l}{2} \\
\delta_{x}(\xi)=\rho(\xi) \frac{q l^{2}}{2 E A}=(2-\xi-\alpha) \xi \frac{q l^{2}}{2 E A}
\end{gathered}
$$

where $\xi=\frac{x}{l}$.

Fig. 3 shows the results of normal effort and tangential displacements normalized for different values of $\alpha$. 


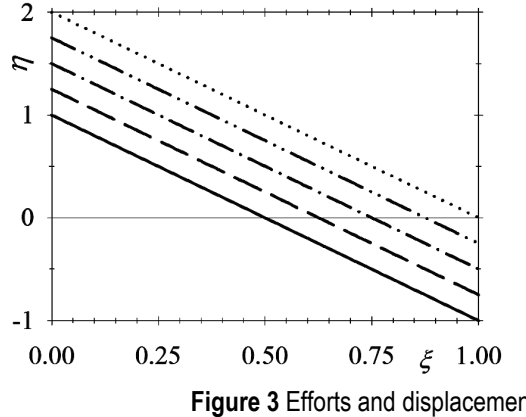

Figure 3 Efforts and displacements of a traction-compression bar with elastic support

\subsection{Circular Helix with Variable Cross-Section}

We present the second example of a helical staircase with a variable circular cross-section, shown in Fig. 4, with a fixed support condition in its basis and free in its upper end.

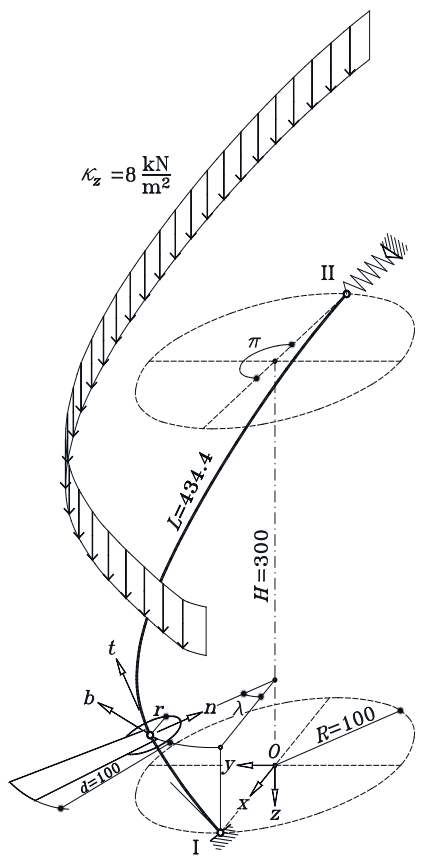

Figure 4 Helical piece with variable circular cross-section

The example is considered to be adequate to show the numerical procedure developed in the article.

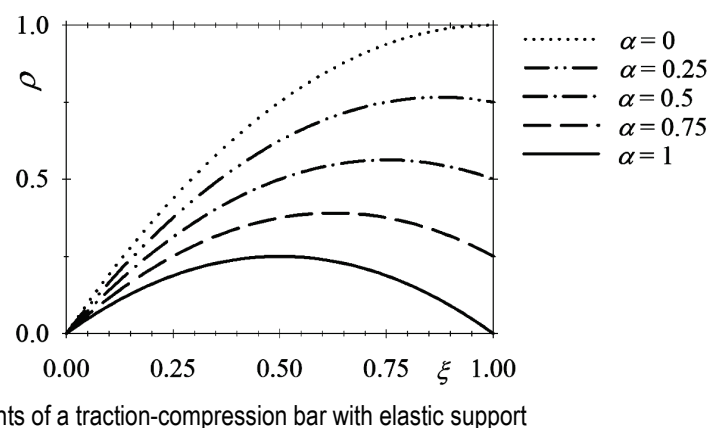

The circular helix, piece that represents the staircase, has a ratio in the basis of $R=100 \mathrm{~cm}$, a height of $H=300$ $\mathrm{cm}$ and a total angle rotated of $\lambda_{f}=\pi \mathrm{rad}$. The crosssection is variable and circular through the axis-line of the helix, of initial ratio $r_{I}=10 \mathrm{~cm}$ and final ratio $r_{I I}=5 \mathrm{~cm}$. Shearing coefficients are $\alpha_{n}=1.33, \alpha_{n b}=0$ and $\alpha_{b}=1.33$. The material of the helical piece is the same through the axis-line (steel), with longitudinal elastic $E=206.01$ $\mathrm{kN} / \mathrm{mm}^{2}$ and transversal elastic $G=79.23 \mathrm{kN} / \mathrm{mm}^{2}$ moduli. The surface load action that is applied in all the length of the stair $d=100 \mathrm{~cm}$ is due to its own weight and its use, considered $\kappa_{z}=8 \mathrm{kN} / \mathrm{m}^{2}$.

This surface load creates two types of actions through the helix axis-line that referred to the general system, which are:

Force action

$q_{x}=0 ; q_{y}=0 ; q_{z}=\kappa_{z} d$

Moment action

$k_{x}=\frac{\kappa_{z} d^{2}}{2} \sin \lambda ; k_{y}=-\frac{\kappa_{z} d^{2}}{2} \cos \lambda ; k_{z}=0$

In this specific problem of the design of a helical staircase of circular variable cross-section, the stiffness matrix $[\boldsymbol{K}]$ and the equivalent action vector $\boldsymbol{q}_{\boldsymbol{k}}$, which are obtained by applying the calculus procedure shown in this article with a number of intervals $n=100$, are:

\begin{tabular}{|c|c|c|c|c|c|c|c|c|c|c|c|}
\hline \multirow{12}{*}{2223.78} & 2259.52 & 2496.91 & 1087.69 & -2081.60 & 801.34 & -2391.78 & 2259.52 & -2336.48 & -520.97 & -1733.16 & -1021.32 \\
\hline & 4301.13 & 4018.42 & 1011.21 & -4849.99 & 4626.54 & -3910.02 & 4301.13 & -2442.30 & -127.68 & -1426.74 & -2314.67 \\
\hline & & 5345.26 & 3299.00 & -7088.75 & 3696.20 & -5224.51 & 4018.42 & -2740.59 & -302.87 & -1431.52 & -1342.42 \\
\hline & & & 4489.23 & -4966.23 & 172.35 & -3245.37 & 1011.21 & -1238.57 & -248.45 & -491.06 & -96.75 \\
\hline & & & & 11588.13 & -5961.00 & 6985.29 & -4849.99 & 2406.07 & 15.16 & 1417.55 & 1313.61 \\
\hline & & & & & 8207.03 & -3655.34 & 4626.54 & -970.82 & -94.04 & -41.60 & -2825.26 \\
\hline & & & & & & 5108.74 & -3910.02 & 2630.01 & 278.54 & 1350.12 & 1293.92 \\
\hline & & & & & & & 4301.13 & -2442.30 & -127.68 & -1426.74 & -2314.67 \\
\hline & $\mathrm{S}$ & $\mathrm{Y}$ & M & & & & & 2460.30 & 534.37 & 1797.29 & 1082.10 \\
\hline & & & & & & & & & 874.21 & 517.97 & -40.02 \\
\hline & & & & & & & & & & 2212.00 & 748.22 \\
\hline & & & & & & & & & & & 2434.69 \\
\hline
\end{tabular}

$\boldsymbol{q}_{\boldsymbol{k}}=\{-17.830,-0.648,-10.962,3.224,12.681,7.568,-13.871,-0.648,-6.817,2.682,-4.532,1.516\}^{\mathrm{T}}$ 
In this case, the fixed support is in the basis and the upper end can change from hinged $(\alpha=1)$ to free $(\alpha=0)$.

This condition is noted as:

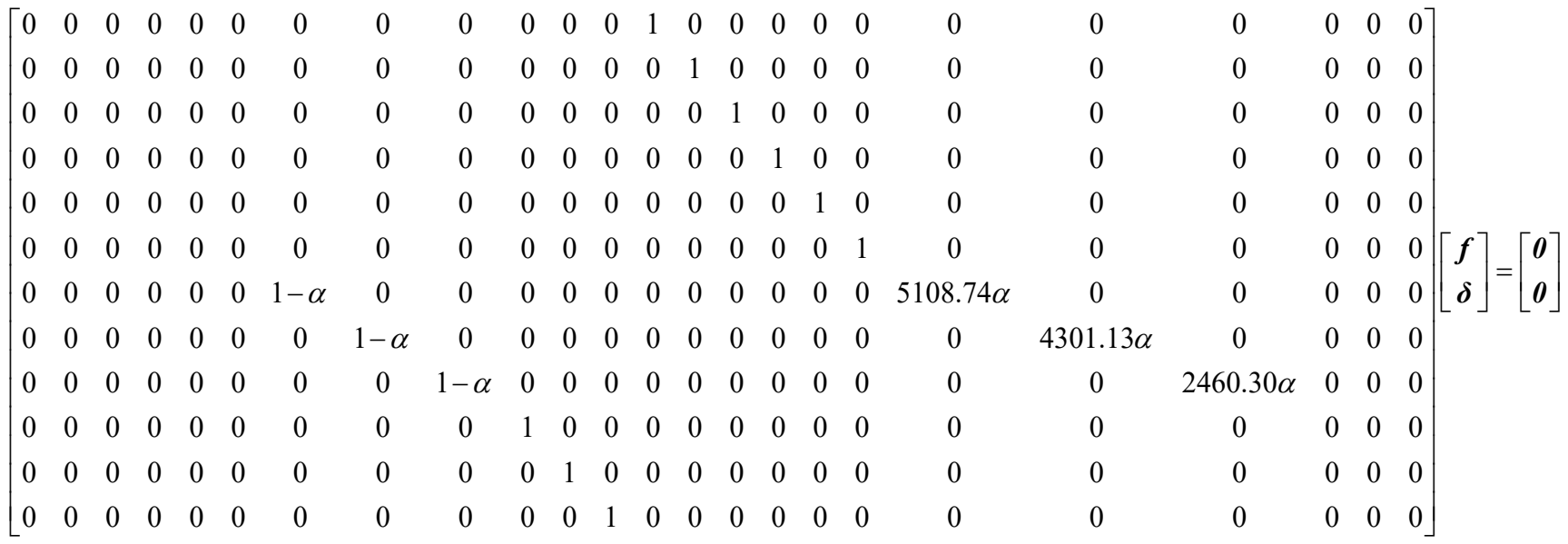

Fig. 5 shows graphics of internal forces and moments for different values of $\alpha(1,0.75,0.5,0.25,0)$ of the helical staircase with circular variable cross-section.
In the same manner, Fig. 6 shows the rotations and displacements for the same values of $\alpha$, used formerly in the helical piece.

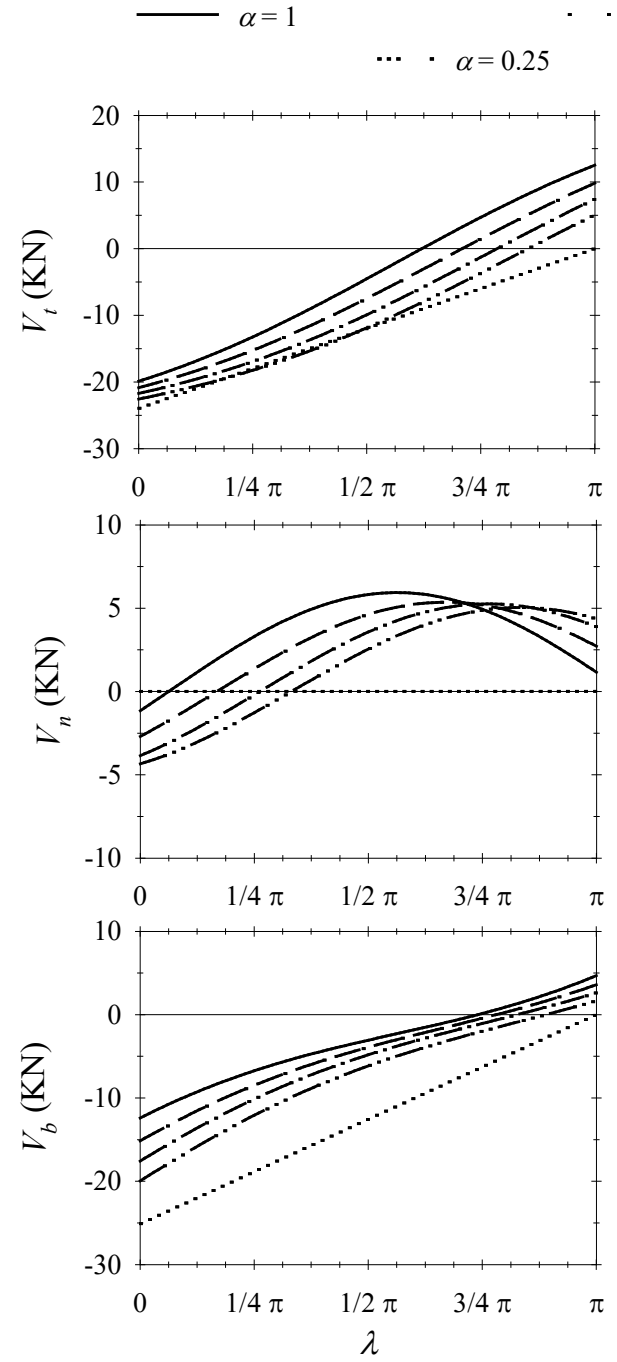

$\alpha=0.75 \quad \alpha=0.5$
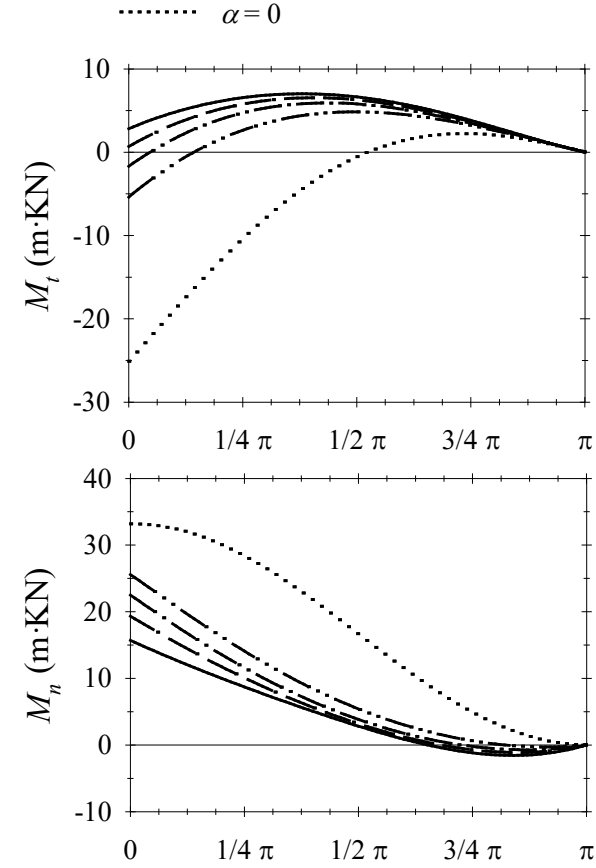

Figure 5 Internal forces and moments of a helical staircase, with variable circular cross-section 


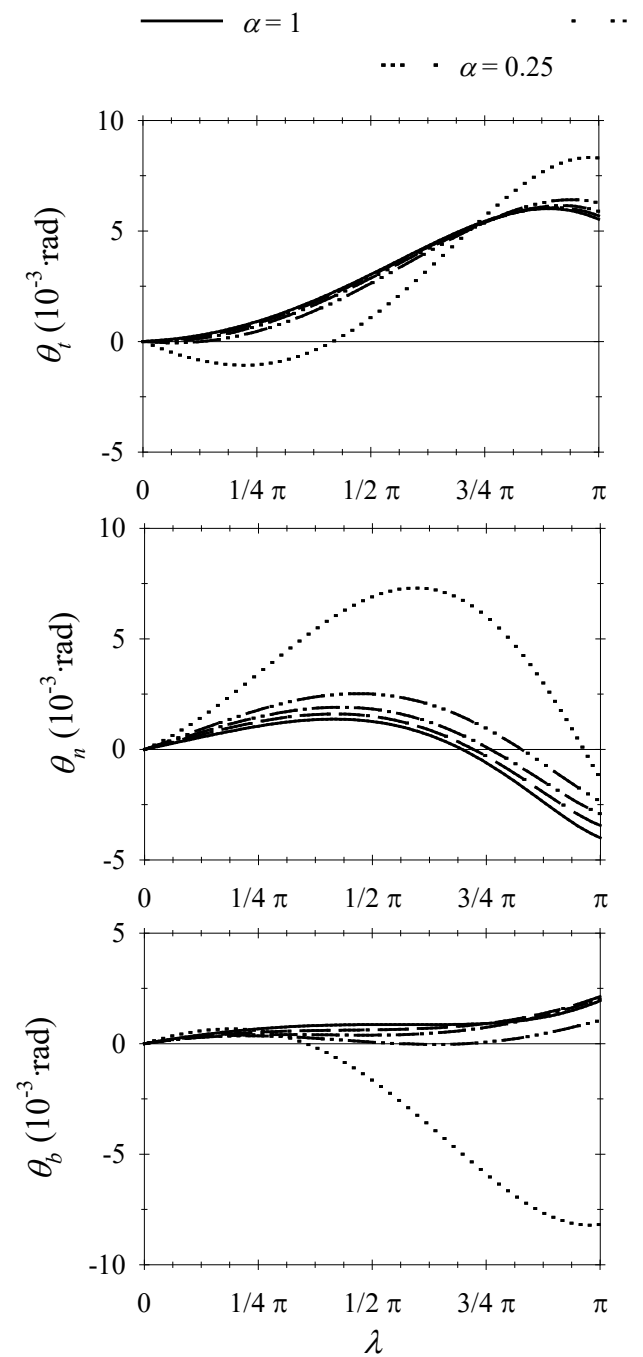

$\alpha=0.75 \quad \alpha=0.5$
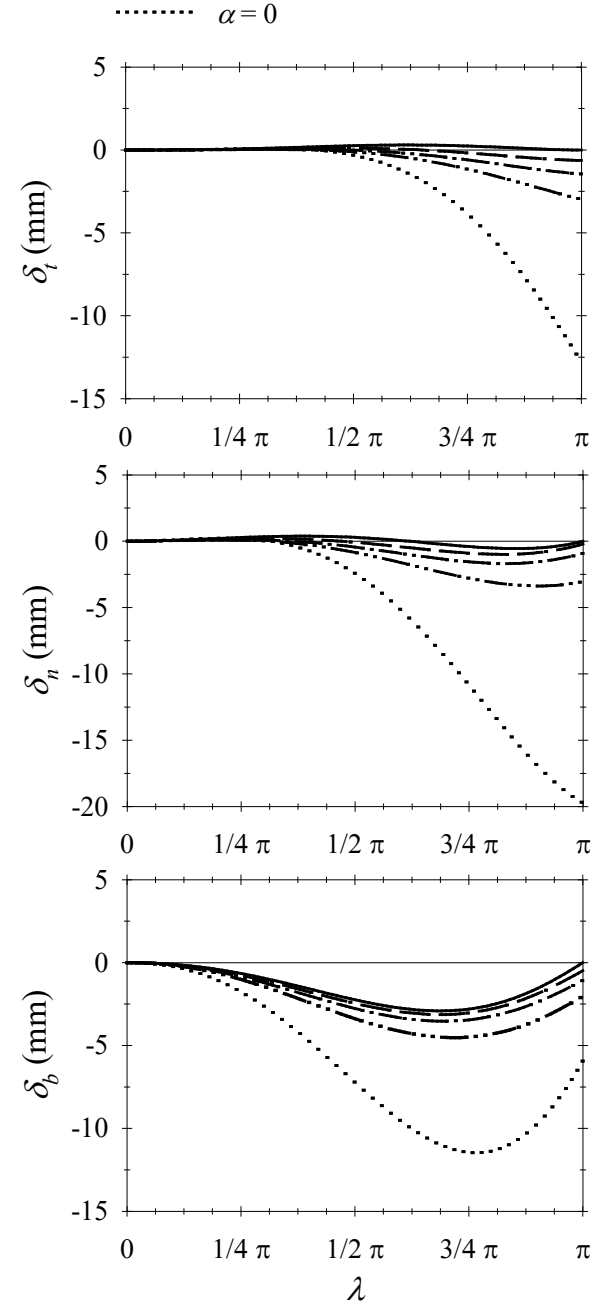

Figure 6 Rotations and displacements of a helical staircase, with variable circular cross-section

\section{CONCLUSIONS}

Establishing the elastic conditions has always been a complicated problem, because usual and traditional models do not contemplate the system with all the functions and all the unknowns. Two examples show good results in practice. This article presents the general system of differential equations that governs the behaviour of a spatially curved beam Eq. (9). This system can be solved by either a numerical or analytical method. The authors present a new numerical process, the Finite Transfer Method, in order to solve a linear system of ordinary differential equations. The way to apply general boundary conditions is presented. It is demonstrated that in the limit, the numerical solution coincides with the exact analytical solution. The Finite Transfer Method seems to be suitable to reach accurate results. The transfer matrix (numerical or exact) is reached directly. When the transfer matrix is rearranged in a new expression, stiffness matrix is derived consequently. Seeing that analytical solution is the limit of the numerical procedure proposed, exact derivations and expressions of transfer and stiffness matrices arise. Let us note that the algebraic system is extended to twenty four equations. This permits the generality of the statement of the problem.

\section{REFERENCES}

[1] Boresi, A. P., Chong, K. P., \& Sagal, S. (2003). Aproximate solution methods in engineering mechanics. John Wiley \& Sons, New Jersey.

[2] Alarcon, E., Brebbia, C., \& Dominguez, J. (1978). The boundary element method in elasticity. Engineering Analysis with Boundary Elements, 20(9), 625-639. https://doi.org/10.1016/0020-7403(78)90021-8

[3] Burden, R. \& Faires, D. (1985). Numerical Analysis. PWS Publishing Company, Boston.

[4] Bathe, K. J. (1996). Finite Element Procedures. Prentice Hall, New Jersey.

[5] Rahman, M. (1991). Applied Differential Equations for Scientists and Engineers: Ordinary Differential Equations. Computational Mechanics Publications, Glasgow.

[4] Bathe, K. J. (1996). Finite Element Procedures. Prentice Hall, New Jersey.

[6] Rajasekaran, S., Gimena, L., Gonzaga, P., \& Gimena, F. N. (2009). Solution method for the classical beam theory using differential quadrature. Structural Engineering Mechanics, 33(6), 675-696. https://doi.org/10.12989/sem.2009.33.6.675

[7] Parcel, J. I. \& Moorman, R. B. (1955). Analysis of statically indeterminate structures. John Wiley, New York.

[8] Yang, Y. B. \& Kuo, S. R. (1987). Effect of curvature on stability of curved beams. Journal of Engineering Mechanics, ASCE, 113(6), 1185-1202. https://doi.org/10.1061/(asce)0733-9445(1987)113:6(1185) 
[9] Washizu, K. (1964). Some considerations on a naturally curved and twisted slender beam. Journal of Applied Mathematics and Physics, 43(2), 111-116. https://doi.org/10.1002/sapm1964431111

[10] Tong, G. \& Xu, Q. (2002). An exact theory for curved beams with any thin-walled open sections. Advances in Structural Engineering, 5(4), 195-209. https://doi.org/10.1260/136943302320974572

[11] Rajasekaran, S. \& Padmanabhan, S. (1989). Equations of curved beams. Journal of Engineering Mechanics, ASCE, 115(5), 1094-1111. https://doi.org/10.1061/(asce)0733-9399(1989)115:5(1094)

[12] Timoshenko, S. (1957). Strength of materials. D. Van Nostrand Company, New York.

[13] Love, A. E. H. (1944). A Treatise on the mathematical theory of elasticity. Dover, New York.

[14] Weiss, H. (2002). Dynamics of Geometrically Nonlinear Rods: I. Mechanical Models and Equations of Motion. Nonlinear Dynamics, 30(4), 357-381. https://doi.org/10.1260/136943302320974572

[15] Moris, D. L. (1968). Curved beam stiffness coefficients. Journal of Structural Division, 94(5), 1165-1178.

[16] Kardestuncer, H. (1974). Elementary matrix analysis of structures. McGraw-Hill, New York.

[17] Leontovich, V. (1959). Frames and arches; condensed solutions for structural analysis. McGraw-Hill, New York.

[18] Molari, L. \& Ubertini, F. (2006). A flexibility-based model for linear analysis of arbitrarily curved arches. / International Journal of Numerical Methods in Engineering, 65(8), 1333-1353. https://doi.org/10.1002/nme.1497

[19] Yamada, Y. \& Ezawa, Y. (1977). On curved finite elements for the analysis of curved beams. International Journal of Numerical Methods in Engineering, 11(11), 635-651. https://doi.org/10.1002/nme.1620111102

[20] Saleeb, A. F. \& Chang, T. Y. (1987). On the hybrid-mixed formulation C0 curved beam elements. Computer Methods in Applied Mechanics and Engineering, 60(1), 95-121. https://doi.org/10.1016/0045-7825(87)90131-9

[21] Just, D. J. (1982). Circularly curved beams under plane loads. Journal of Structural Division, 108(8), 1858-1873.

[22] Shi, G. \& Voyiadjis, G. Z. (1991). Simple and efficient shear flexible two-node arch/beam and four-node cylindrical shell/plate finite elements. International Journal of Numerical Methods in Engineering, 31(4), 759-776. https://doi.org/10.1002/nme.1620310408

[23] Lee, H. P. (1969). Generalized stiffness matrix of a curvedbeam element. AIAA Journal, 7(10), 2043-2045. https://doi.org/10.2514/3.5513

[24] Benedetti, A. \& Tralli, A. (1989). A new hybrid F.E. model for arbitrarily curved beam-I. Linear analysis. Computers and Structures, 33(6), 1437-1449. https://doi.org/10.1016/0045-7949(89)90484-7

[25] Wang, T. M. \& Merrill, T. F. (1988). Stiffness coefficients of noncircular curved beams. Journal of Structural Engineering, 114(7), 1689-1699. https://doi.org/10.1061/(asce)0733-9445(1988)114:7(1689)

[26] Gimena, L., Gonzaga, P., \& Gimena, F. N. (2010). Forces, moments, rotations, and displacements of polynomialshaped curved beams. International Journal of Structures Stability and Dynamics, 10(1), 77-89. https://doi.org/10.1142/s0219455410003336

[27] Marquis, J. P. \& Wang, T. M. (1989). Stiffness matrix of parabolic beam element. Computers and Structures, 31(6), 863-870. https://doi.org/10.1016/0045-7949(89)90271-X

[28] Scordelis, A. C. (1960). Internal forces in uniformly loaded helicoidal girders. Journal of the American Concrete Institute, 31(4), 1013-1026. https://doi.org/10.14359/8127

[29] Gimena, F. N., Gonzaga, P., \& Gimena, L. (2008). 3Dcurved beam element with varying cross-sectional area under generalized loads. Engineering Structures, 30(2), 404-411. https://doi.org/10.1016/j.engstruct.2007.04.005

[30] Gimena, L., Gonzaga, P., Gimena, F. N. (2014). Boundary equations in the finite transfer method for solving differential equation systems. Applied Mathematical Modelling, 38(9-10) 2648-2660. https://doi.org/10.1016/j.apm.2013.11.001

[31] Sokolnikoff, I. S. \& Redeffer, R. M. (1958). Mathematics of Physics and Modern Engineering. McGraw-Hill, Tokyo. https://doi.org/10.1149/1.2428901

[32] Timoshenko, S. (1953). History of strength of materials. McGraw-Hill, New York.

[33] Gimena, L., Gimena, F. N., \& Gonzaga, P. (2008) Structural analysis of a curved beam element defined in global coordinates. Engineering Structures, 30(11), 33553364. https://doi.org/10.1016/j.engstruct.2008.05.011

[34] Gimena, F. N., Gonzaga, P., \& Gimena, L. (2009). Numerical transfer-method with boundary conditions for arbitrary curved beam elements. Engineering Analysis with Boundary Elements, 33(2), 249-257. https://doi.org/10.1016/j.enganabound.2008.04.004

[35] Gonzaga, P., Gimena, F. N., \& Gimena, L. (2014). Stiffness and transfer matrix analysis in global coordinates of a 3D curved beam. International Journal of Structural Stability and Dynamics, 6(7), 19 pages. https://doi.org/10.1142/s0219455414500199

\section{Contact information:}

Fernando SARRIA, PhD researcher

Public University of Navarre

Tejos building, Campus Arrosadia

31006 Pamplona, Spain

E-mail: fsarria@fsestructuras.com

Faustino N. GIMENA, Professor

Public University of Navarre

Tejos building. Campus Arrosadia

31006 Pamplona, Spain

E-mail: faustino@unavarra.es

Pedro GONZAGA, Professor

Public University of Navarre

Tejos building, Campus Arrosadia

31006 Pamplona, Spain

E-mail: pedro.gonzaga@unavarra.es

Mikel GOÑI, Professor

Public University of Navarre

Tejos building, Campus Arrosadia

31006 Pamplona, Spain

E-mail: mikel.goni@unavarra.es

Lázaro GIMENA, Professor

Public University of Navarre

Tejos building, Campus Arrosadia

31006 Pamplona, Spain

E-mail: lazaro.gimena@unavarra.es 